\title{
TERAPIA DE INDUÇÃO COM TIMOGLOBULINA VERSUS ANTICORPO MONOCLONAL ANTI - IL2R - UMA ANÁLISE PAREADA EM TRANSPLANTE RENAL COM DOADOR FALECIDO
}

\author{
Deceased kidney transplantation with thymoglobulin versus anti-IL2R monoclonal antibody \\ induction - A paired kidney analysis
}

\author{
Cláudia Maria Costa de Oliveira, ${ }^{12}$, Diego Morais Gomes', Camilo Reuber ${ }^{2}$, Daniela Costa de Oliveira Santos ${ }^{1}$, Maria Luisa Mattos Brito \\ Oliveira², Paula Castelo Branco Fernandes', João Evangelista Júnior', Ronaldo Matos Esmeraldo²
}

\begin{abstract}
RESUMO
Introdução: A timoglobulina é um anticorpo policlonal utilizado na terapia de indução em transplante (Tx) renal e que tem sido associado à redução na incidência de função retardada do enxerto (DGF) e rejeição aguda. Objetivo: Comparar terapia de indução com timoglobulina versus anticorpos monoclonais anti-IL2R em uma análise pareada de transplantes renais com doador falecido. Métodos:Todos os rins pareados de doador falecido no período de 2006-2008, onde um dos rins foi alocado para um paciente recebendo timoglobulina (grupo 1) e o seu par foi alocado para um paciente recebendo anticorpo monoclonal anti-IL2R (grupo 2) foram incluídos neste estudo retrospectivo. As taxas de DGF e rejeição aguda, a função renal e a incidência de doença por citomegalovírus (CMV), no primeiro ano de transplante, foram avaliadas. Resultados: Foram incluídos 94 receptores, idade média 38,4 anos, 60,6 \% do sexo masculino e tempo médio em diálise 4,11 anos. Não houve diferença estatística entre os dois grupos em relação à idade e sexo do receptor, número de mismatches HLA, anticorpos reativos contra painel (PRA) e tempo de isquemia fria (TIF). O protocolo imunossupressor inicial consistiu de micofenolato mofetil e inibidor de calcineurina (tacrolimus: 88,3\%) e prednisona em $62 \%$ dos pacientes. Em relação aos doadores, $63,8 \%$ eram do sexo masculino, idade média 30,9 anos, creatinina sérica $1,17 \mathrm{mg} / \mathrm{dl}$, causas da morte encefálica: traumatismo crânio-encefálico em $48,9 \%$ e acidente vascular cerebral em 19,1\%. A função renal avaliada pela creatinina aos três, seis e doze meses pós-Tx, a incidência de DGF (56,6 vs 63,0\%) e de CMV doença (10,9 vs 15,2\%) não foi estatisticamente diferente entre os grupos. A taxa de rejeição aguda foi significativamente menor no grupo da timoglobulina $(19,6$ vs 45,7\%; $p=0,008)$. Conclusão: A terapia de indução com timoglobulina associou-se com uma redução significativa na incidência de rejeição aguda no primeiro ano de transplante nessa coorte de receptores de rins pareados de doador falecido. Não foi detectada redução na taxa de DGF e não houve impacto na função renal/ incidência de CMV doença com o uso da timoglobulina.
\end{abstract}

Descritores: Transplante Renal; Imunossupressão; Doador falecido; Globulina Antilinfocitária; Anticorpos Monoclonais.

\section{Instituições: \\ 1 Unidade de Transplante Renal do Hospital Universitário Walter Cantídeo - Universi- dade Federal do Ceará (UFC) - Fortaleza, Ceará, Brasil. \\ 2 Unidade de Transplante Renal do Hospital Geral de Fortaleza (HGF) - Fortaleza, Ceará, Brasil}

\section{Correspondência:}

Claudia Maria Costa de Oliveira

Nefrologia, Universidade Federal do Ceará - Brasil

Rua Professor Jacinto Botelho, 500 / 500, CEP 60.810-050, Fortaleza/CE

Tel.: (51) 3241-2036 Fax: (85) 3366-8611

E-mail: claudiadrl@gmail.com

Recebido em: 18.05.2011

Aceito em: 20.06.11

\section{INTRODUÇÃO}

A Timoglobulina é um importante anticorpo policlonal obtido da imunização de coelhos com timócitos humanos, que tem sido utilizado desde 1984 em transplante de órgãos para prevenção e tratamento da rejeição aguda, inclusive rejeição esteróideresistente ${ }^{1}$ e também para permitir a introdução mais tardia dos inibidores de calcineurina ${ }^{2,3}$ A timoglobulina tem sido associada à redução na incidência de rejeição aguda e função retardada do enxerto (DGF), ${ }^{4,5}$ embora os resultados descritos na literatura sejam variáveis, dependendo da população estudada. ${ }^{6,7}$

O mecanismo de ação da timoglobulina envolve a depleção rápida de células $\mathrm{T}$, através da lise celular dependente de complemento e morte de linfócitos apoptóticos. ${ }^{8}$ Além do efeito de depleção de células $T$, a timoglobulina também modula marcadores de superfície celular, incluindo integrinas e moléculas de adesão intercelular que facilitam a adesão de leucócitos ao endotélio. ${ }^{8}$ Esse efeito da timoglobulina pode ser responsável pela atenuação da injúria isquemia-reperfusão em rins transplantados. ${ }^{9}$

A injúria isquemia-reperfusão associa-se com maior taxa de rejeição aguda, função retardada do enxerto (DGF), falência tardia e 
perda do enxerto. ${ }^{10,11}$ Portanto, a injúria isquemia-reperfusão tem um importante papel na qualidade e na função do enxerto e é uma importante causa para maior tempo de hospitalização e menor sobrevida do enxerto em longo prazo. ${ }^{12}$

Os efeitos adversos do uso da timoglobulina incluem febre, anormalidades hematológicas, aumento na incidência de infecções, especialmente por citomegalovírus (CMV), e em alguns estudos o aumento na incidência de doença linfoproliferativa pós-Tx. ${ }^{13}$

O basiliximab é um anticorpo monoclonal quimérico (humano/ murino), antagonista do receptor de IL2 (IL2RA) em linfócitos T ativados que também reduz significativamente a taxa de rejeição aguda em transplante renal. ${ }^{14}$ Três metanálises confirmam a eficácia do basiliximab na prevenção da rejeição aguda em Tx renal quando comparado ao placebo. ${ }^{14-16} \mathrm{~A}$ incidência de efeitos adversos foi similar no grupo de pacientes em uso de basiliximab ou placebo, sem aumento na incidência de infecção por CMV ou malignidades.

Como os custos e efeitos colaterais das duas classes de medicamentos são diferentes, os benefícios da utilização da Timoglobulina em termos de eficácia, toxicidade e custos precisa ser melhor definido.

Os autores realizaram uma análise pareada de transplantes renais com doador falecido para comparar as terapias de indução com timoglobulina versus basiliximab, na prevenção de rejeição aguda e função retardada do enxerto, na prevalência de doença por citomegalovírus e na função renal em 12 meses pós-transplante. A estratégia de análise pareada pode minimizar a variabilidade do doador e tem sido utilizada em poucos estudos. Na prática clinica, em razão da diferença no custo e no perfil de eventos adversos e da semelhança na eficácia das drogas, não há consenso sobre qual terapia de indução deve ser preferida.

\section{MÉTODOS}

Os autores realizaram um estudo de coorte retrospectivo, em que foram incluídos todos os receptores de transplante renal de dois hospitais de Fortaleza que receberam rins pareados de doador falecido no período de 2006-2008, onde um dos rins foi alocado para um paciente recebendo terapia de indução com TIMOGLOBULINA em um hospital (grupo 1) e seu par foi alocado para um paciente recebendo BASILIXIMAB em um segundo hospital (grupo 2). As equipes de transplante dos hospitais 1 e 2 eram diferentes e seguiam protocolos de imunossupressão diferentes, com indução preferencial com timoglobulina no hospital 1 e com basiliximab no hospital 2, na época em que os pacientes foram selecionados. Todos os transplantes com doador falecido realizados no período em estudo foram identificados nos dois hospitais, sendo incluídos apenas os transplantes em que um dos rins tivesse sido alocado em um hospital e o outro rim do mesmo doador, no segundo hospital. Os prontuários médicos e as fichas de seguimento ambulatorial pós-transplante foram pesquisados, sendo os dados dos pacientes avaliados desde a realização do transplante até 12 meses póstransplante (pós-Tx).

A ocorrência de função retardada do enxerto e rejeição aguda, a função renal avaliada pela creatinina sérica e a incidência de doença por citomegalovírus (CMV) no primeiro ano de Tx foram avaliadas nos pacientes pareados pelo doador falecido.

Função retardada do enxerto foi definida como a necessidade de diálise na primeira semana pós-transplante. Os casos de rejeição aguda foram confirmados histologicamente, tendo sido ainda considerados como rejeição aguda os casos com alterações borderline à biópsia do enxerto e que foram tratados com pulsoterapia com metilprednisolona. A doença por CMV foi definida como os casos com suspeita clínica que tinham sido tratados com ganciclovir e, posteriormente, confirmados com PCR para CMV ou sorologia para CMV IgM positiva.

As variáveis contínuas foram comparadas pelo teste $t$ de Student e as variáveis categóricas pelo teste de qui-quadrado/Fisher. Um valor de $\mathrm{p}<0,05$ foi considerado estatisticamente significante.

Os dados foram registrados em uma planilha de Microsoft Excel 5.0 e depois transferidos para o programa SPSS versão 10.0 (Incorporation Statiscal Package for the Social Science for Windows Student version) onde a análise estatística foi realizada.

O presente projeto foi submetido ao Comitê de Ética em Pesquisa em Seres Humanos dos dois hospitais que incluíram pacientes, em conformidade com a resolução $n^{\circ}$ 196/96 do Conselho Nacional de Saúde/ Ministério da Saúde

\section{RESULTADOS}

Foram realizados 94 transplantes a partir de 47 doadores falecidos no período do estudo, sendo $60,6 \%$ dos receptores do sexo masculino, com idade média de $38,4 \pm 12,9$ anos e com tempo médio em diálise de 4,11 $\pm 3,25$ anos. As características demográficas dos receptores e doadores em estudo encontram-se na Figura 1. Podemos observar que são doadores jovens (com idade média de 31 anos) e o traumatismo crânio-encefálico (TCE) é a principal causa de morte, o que representa uma do grupo de doadores de baixo risco (Figura 1).

Figura 1. Características demográficas da população em estudo

\begin{tabular}{|ll|}
\hline N. de pacientes & 94 \\
Gênero do receptor & Masculino: $57(60,6 \%)$ \\
Idade média do receptor & $38,4 \pm 12,9$ anos \\
$1^{0}$ transplante & $91(96,8 \%)$ \\
Tempo médio em diálise & $4,11 \pm 3,25$ anos \\
CMV IgG + & Receptor: $90,9 \%$ Doador: $88,3 \%$ \\
Gênero do doador & Masculino: $60(63,8 \%)$ \\
Idade média do doador & $30,9 \pm 14,6$ anos \\
Creatinina do doador & $1,17 \pm 0,48$ mg/dl \\
Causa mortis do doador & TCE: $48,9 \%$ AVC: $19,1 \%$ \\
\hline
\end{tabular}

O protocolo imunossupressor utilizado nos dois hospitais encontrase na Figura 2, sendo que 38,3\% do total de pacientes avaliados não utilizaram corticóide na terapia de manutenção ( 57,5 \% no hospital 1 e 19,2\% no hospital 2).

Figura 2. Protocolo imunossupressor utilizado na população em estudo.

\begin{tabular}{|ll|}
\hline Ciclosporina & $08(8,5 \%)$ \\
Tacrolimus & $83(88,3 \%)$ \\
Micofenolato sódico & $43(45,8 \%)$ \\
Micofenolato mofetil & $51(54,2 \%)$ \\
Prednisona* & $58(61,7 \%)$ \\
\hline
\end{tabular}

${ }^{*} 42,5 \%$ dos pacientes no grupo 1 e $80,8 \%$ no grupo 2 
Os dados referentes aos receptores e à evolução pós-transplante nos dois hospitais foram comparados na Tabela 1. Não houve diferença estatisticamente significante entre os dois grupos em relação à idade e ao sexo do receptor, tempo em diálise, número de mismatches HLA (Human Leukocyte Antigen), anticorpos reativos contra o painel de linfócitos (PRA), tempo de isquemia fria (TIF).

Tabela 1. Avaliação de variáveis do transplante e da evolução pós-transplante na população em estudo, segundo a terapia de indução utilizada.

\begin{tabular}{llll} 
Variável & $\begin{array}{c}\text { Timoglobulina(grupo1) } \\
\mathbf{n = 4 7}\end{array}$ & $\begin{array}{c}\text { Basiliximab(grupo2) } \\
\mathbf{n = 4 7}\end{array}$ & $\mathbf{p}$ \\
\hline Idade do receptor & $39,04 \pm 12,82$ & $38,57 \pm 13,50$ & 0,864 \\
Sexo do receptor (Masc) & $59,5 \%$ & $61,7 \%$ & 0,833 \\
Mismatches HLA & $4,21 \pm 1,01$ & $4,27 \pm 0,76$ & 0,855 \\
Tempo isquemia fria (hs) & $23,7 \pm 3,26$ & $23,3 \pm 3,63$ & 0,677 \\
DGF & $56,5 \%$ & $63,0 \%$ & 0,524 \\
Tempo médio de DGF & $16,0 \pm 10,82$ & $13,73 \pm 8,93$ & 0,543 \\
Rejeição aguda** & $19,6 \%$ & $45,7 \%$ & 0,008 \\
RA maior que borderline & $10,8 \%$ & $23,4 \%$ & 0,227 \\
CMV doença & $10,9 \%$ & $15,2 \%$ & 0,535 \\
Creat. alta hospitalar* & $2,62 \pm 1,56$ & $1,96 \pm 1,21$ & 0,021 \\
Creat. 3 meses pós-Tx & $1,77 \pm 1,01$ & $1,77 \pm 1,74$ & 0,216 \\
Creat. 6 meses pós-Tx & $1,53 \pm 0,92$ & $1,51 \pm 1,07$ & 0,441 \\
Creat. 12 meses pós-Tx & $1,52 \pm 0,76$ & $1,37 \pm 0,66$ & 0,261 \\
& & &
\end{tabular}

Creat. $=$ creatinina

$\mathrm{RA}=$ rejeição aguda

${ }^{* *} p<0,05$

O tempo de isquemia fria foi de $23,7 \pm 3,26$ horas no hospital $1 \mathrm{e}$ $23,3 \pm 3,36$ horas no hospital $2(\mathrm{p}=\mathrm{NS})$. A prevalência de DGF foi de $56,5 \%$ no hospital 1 e $63 \%$ no hospital $2(p=N S)$ e o tempo médio de hemodiálise nos pacientes que complicaram com DGF foi de $16,0 \pm 10,8$ dias no hospital 1 e 13, $73 \pm 8,93$ dias no hospital $2(\mathrm{p}=\mathrm{NS})$.

Uma diferença significativa foi observada na prevalência de rejeição aguda, que foi significativamente mais alta no grupo que recebeu basiliximab como terapia de indução e na creatinina na alta hospitalar, que foi menor no mesmo grupo.

As alterações borderline na biópsia renal foram incluídas como rejeição aguda. Uma segunda análise após a exclusão desses casos (que representaram quatro casos no grupo 1 e 10 casos no grupo 2), demonstrou uma incidência de rejeição aguda de $10,8 \%$ no grupo 1 e $23,4 \%$ no grupo $2(\mathrm{p}=\mathrm{NS})$.

\section{DISCUSSÃO}

A população do presente estudo compreendeu um grupo de receptores jovens (38 anos em média) e de doadores jovens (31 anos em média), com TCE como a principal causa de morte encefálica $(48,9 \%)$ e com função renal bem preservada no momento da doação (creatinina de $1,17 \mathrm{mg} / \mathrm{dl}$ ). Uma característica especial foi que o grupo que fez uso de timoglobulina, 57,5\% não usou esteróides na terapia de manutenção, diferentemente do grupo que fez uso de basiliximab. Uma vantagem do uso da timoglobulina é que o seu uso em combinação com terapia de manutenção sem esteróides tem sido eficaz na prevenção de rejeição aguda em longo prazo em receptores adultos e pediátricos. ${ }^{17}$

Embora a população de doadores fosse considerada de baixo risco, observamos um tempo de isquemia fria prolongado nos dois hospitais (23,7 e 23,3 horas) e uma elevada prevalência de função retardada do enxerto (56,5 e 63\%), o que pode ter traduzido problemas na manutenção do doador antes da captação dos órgãos. Apesar do uso da timoglobulina, que permitiu a introdução retardada dos inibidores de calcineurina no grupo 1 , não houve diferença significativa na prevalência e na duração da função retardada do enxerto (16 vs 13,7 dias), o que pode ter sido determinado pelo tempo de isquemia fria prolongado, manutenção do doador de má qualidade ou tamanho pequeno da amostra em estudo, que não permitiu que uma diferença fosse observada.

Foi previamente demonstrado que a função retardada do enxerto tem um significante impacto na sobrevida em longo prazo do enxerto. ${ }^{18}$ Em pacientes de baixo risco imunológico, a incidência de DGF geralmente não foi diferente entre a timoglobulina (rATG) e o basiliximab ([22\% vs $18 \%]^{19}$ e $\left.[30 \% \text { vs } 29 \%]^{20}\right)$, mas a incidência de DGF foi significativamente menor no grupo da timoglobulina em pacientes de alto risco. ${ }^{6}$

Em relação à prevenção de rejeição aguda, a timoglobulina foi mais eficaz do que o basiliximab na população do presente estudo (19,6 vs 45,7\%). Em pacientes de baixo risco imunológico, foram realizados quatro pequenos estudos prospectivos, randomizados e controlados, comparando timoglobulina e IL2RA como terapia de indução, ${ }^{19-22}$ sendo pacientes de baixo risco imunológico os transplantes primários e pacientes com PRA $<25 \%$. De um modo geral, nesses estudos a incidência de rejeição aguda confirmada por biópsia foi semelhante nos dois grupos, não houve diferença na sobrevida do enxerto ou dos pacientes aos seis e doze meses pós-Tx e o uso de timoglobulina foi associado com mais efeitos adversos. Contudo, os estudos comparando timoglobulina e basiliximab geralmente não tinham poder suficiente para mostrar a superioridade de um regime em relação ao outro. ${ }^{19}$

No presente estudo, quando retiramos da análise os pacientes com diagnóstico de alterações borderline que foram tratados como rejeição aguda, não houve diferença significativa na taxa de rejeição aguda. Entretanto, não podemos afirmar se hão havia maiores graus de rejeição, uma vez que muitas vezes temos problemas de amostragem nos fragmentos de biópsia renal e a rejeição aguda é um processo esparso. Um diagnóstico de alterações borderline pode em alguns casos representar um grau maior de rejeição aguda não aparente no fragmento analisado.

Dois estudos prospectivos, randomizados, em pacientes de alto risco para Tx renal, demonstraram uma vantagem da indução com timoglobulina comparados aos anticorpos monoclonais. ${ }^{6,7} \mathrm{~A}$ timoglobulina foi mais eficaz do que o daclizumab na prevenção de rejeição aguda em receptores de Tx renal com alto risco de rejeição aguda em terapia tríplice com MMF.

Mandelbrot ${ }^{23}$ sugeriu um algoritmo para o uso de IL2RA em pacientes de baixo risco imunológico e Timoglobulina em pacientes de alto risco. Essa recomendação é consistente com as diretrizes KDIGO (Clinical Practice guidelines for the care of the Transplant recipient). ${ }^{24}$ De acordo com as diretrizes KDIGO, o uso de timoglobulina em pacientes de alto risco para rejeição aguda supera os seus potenciais riscos.

Em receptores idosos ( $>60$ anos), a timoglobulina pode ser a terapia de indução de escolha em receptores de alto risco com doadores 
Cláudia Maria Costa de Oliveira, Diego Morais Gomes, Camilo Reuber, Daniela Costa de Oliveira Santos, Maria Luisa Mattos Brito Oliveira, Paula Castelo Branco Fernandes,

João Evangelista Júnior, Ronaldo Matos Esmeraldo

de alto risco e possivelmente receptores de baixo risco com doadores de alto risco. ${ }^{25}$ Receptores de alto risco imunológico foram definidos como aqueles com PRA $>20 \%$ antes do Tx renal ou raça negra. Doadores de alto risco foram aqueles com critérios expandidos de doação, doadores após a parada cardíaca e com tempo de isquemia fria $>24$ horas. ${ }^{25}$

Em relação à função renal avaliada pela creatinina sérica, a mesma não foi diferente aos três, seis e doze meses pós-Tx renal nos dois grupos em estudo. Em estudos prévios, também não foi evidenciada diferença significativa entre a timoglobulina e o basiliximab em relação aos níveis de creatinina, até um ano pós-Tx, exceto com quatro semanas de $\mathrm{Tx}{ }^{19}$ e não houve diferença na sobrevida do enxerto/paciente e na função renal após um ano medida pela creatinina e taxa de filtração glomerular estimada. ${ }^{7}$

Não houve diferença na prevalência de citomegalovírus doença no primeiro ano pós-Tx renal no presente estudo (10,9 vs $15,2 \%)$, mas deve ser observado que todos os pacientes do grupo da timoglobulina fizeram profilaxia com ganciclovir por três meses, diferentemente do grupo do basiliximab (com exceção dos receptores CMV negativo, com doador CMV positivo $(\mathrm{n}=4)$ ). Não podemos afirmar que não houve diferença na prevalência de citomegalovírus infecção, pois não foi realizada monitorização da replicação viral através de PCR para CMV ou antigenemia a intervalos regulares pós-Tx renal.

É limitação do presente estudo o fato de que, além da terapia de indução diferente nos dois grupos em estudo, o uso de esteróides na terapia de manutenção teve uma prevalência diferente entre os grupos $(42,5 \%$ no grupo 1 vs $80,8 \%$ no grupo 2$)$. Isso poderia teoricamente ter sido um viés no estudo, mas o número pequeno de pacientes não permitiu a análise estatística em quatro grupos (com e sem timoglobulina e com e sem esteróides).

Estudos com maior número de pacientes e com seguimento mais longo, com o objetivo de avaliar a eficácia da timoglobulina em relação ao basiliximab, devem ser realizados para definir melhor o papel da timoglobulina em relação aos anticorpos monoclonais. A timoglobulina pode representar uma importante ferramenta para aumentar o pool de doadores, ao aumentar a possibilidade de utilização de doadores limítrofes.

\section{CONCLUSÃO}

No presente estudo, a terapia de indução com timoglobulina associou-se a uma redução significativa na incidência de rejeição aguda no primeiro ano de Tx, quando comparada ao basiliximab em transplante renal com doadores pareados, mas não foi detectada uma redução significativa na taxa de DGF, provavelmente em função do TIF prolongado nos dois grupos.

Não houve impacto na função renal e na incidência de CMV doença no primeiro ano de Tx com o uso de timoglobulina, apesar da redução na taxa de rejeição aguda.

Estudos adicionais, com maior número de pacientes e utilizando a mesma estratégia de avaliação de transplantes com doadores pareados, são necessários para identificar qual grupo de receptores/ doadores apresentam maior benefício com a indicação do uso da terapia de indução com timoglobulina.

\section{ABSTRACT:}

Purpose: Thymoglobulin is an important polyclonal antibody used in kidney transplantation as an induction therapy, and it has been associated to a decrease in delayed graft function and acute rejection rates. The authors used a paired kidney analysis to compare thymoglobulin with other induction therapies, in order to minimize the donor variabilility and bias. Methods: All paired kidneys from deceased donors during the period 2006-2008, where one kidney was allocated to a patient receiving thymoglobulin (group1) and its mate allocated to a patient receiving anti IL-2R monoclonal antibodies (group 2) were assessed in this retrospective study. To investigate the impact of induction therapy, we investigated the delayed graft function rates (DGF), acute rejection (AR) and renal function in the first year after the transplant (Tx) in the paired kidney subjects. Results: A total of 94 Txs were investigated: mean age: 38.4 years, being $60.6 \%$ male and with average dialysis time of 4.11 yrs. No statistically significant difference was found between both groups in relation to the recipient age, gender, HLA mismatches, PRA, cold ischemia time (CIT). Immunosuppressive protocol consisted to mofetil mycophenolate plus calcineurin inhibitors (tacrolimus in $88.3 \%$ of patients) in both groups, and steroids in $62 \%$ of patients. Deceased donor characteristics were: $63.8 \%$ male, mean age $30.9 \mathrm{ys}$, serum creatinine $1.17 \mathrm{mg} / \mathrm{dl}$. The most important causes of death were trauma in $48.9 \%$ and cerebrovascular accident in $19.1 \%$ donors. The magnitude of renal function at 3, 6 and 12 post-Tx months and the DGF rate $(56.6 \%$ vs $63.0 \%)$ were not statistically different between both groups. The rate of any AR report within 1 post-Tx year was significantly lower in the Thymo group $(19.6 \mathrm{vs} 45.7 \%$; $\mathrm{p}=0.008)$, and the incidence of CMV disease was similar in both groups (10.9 vs $15.2 \% ; p=0.535)$. Conclusion: Thymoglobulin was associated to a significant reduction in $1 \mathrm{yr}$-acute rejection rate in this cohort of paired kidneys, but without presenting a decreased DGF rate. There was no impact on renal function/CMV disease in the 1st post-Tx year.

Keywords: Kidney Transplantation; Immunosuppression; Deceased Donor. Antilymphocyte globulin; Antibodies, Monoclonal 


\section{REFERÊNCIAS:}

1. Cosimi AB. Antilymphocyte globulins and monoclonal antibodies. In: Morris PJ, ed. Kidney Transplantation: Principles and Pratice. Philadelphia: Saunders; 1988. p. 343. 2. Wiland AM, Fink JC, Weir MR, Philosophe B, Blahut S, Weir MRJ, et al. Should living-unrelated renal transplant recipients receive antibody induction? Results of a clinical experience trial. Transplantation. 2004;77(3):422-5.

3. Peddi VR, Bryant M, Roy-Chaudhury P, Wodle ES, First MR. Safety, efficacy and cost analysis of Thymoglobulin induction therapy with intermittent dosing based on CD3+ lymphocyte counts in kidney and kidney-pancreas transplant recipients. Transplantation. 2002;73(9):1514-8.

4. Deeks ED, Keating GM. Rabbit antithymocyte globulin (thymoglobulin): a review of its use in the prevention and treatment of acute renal allograft rejection. Drugs. 2009;69 (11):1483-512. 5. Gaber AO, Knight RJ, Patel S, Gaber LW. A review of the evidence for use of thymoglobulin induction in renal transplantation. Transplant Proc. 2010;42 (5):1395-400.

6. Brennan DC, Daller JA, Lake KD, Cibrik D, Del Castillo D. Rabbit antithymocyte globulin versus basiliximab in renal transplantation. N Engl J Med. 2006;355(19):1967-77.

7. Noel C, Abramowicz D, Durand D, Mourad G, Lang P, Kessler M, et al. Daclizumab versus antithymocyte globulin in high-immunological-risk renal transplant recipients. J Am Soc Nephrol. 2009;20(6):1385-92.

8. Mueller TF. Mechanisms of action of thymoglobulin. Transplantation. 2007;84(11S): S5-S10.

9. Moura LRR, Pereira MG, Durão M, Tonato EJ, Matos ACC, Wroclawsky ER, et al. Thymoglobulin as an induction theraphy: protection against ischemia and reperfusion injury. Einstein. 2006;4(4):315-20.

10. Goggins WC, Pascual MA, Powelson JA, Magee C, Tolkoff-Rubin N, Farrel $\mathrm{ML}$, et al. A prospective, randomized, clinical trial of intraoperative versus postoperative thymoglobulin in adult cadaveric renal transplant recipients. Transplantation. 2003;76(5):798-802.

11. Bogetti D, Sankary HN, Jarzembowski TM, Manzelli A, Knight PS, Thielke $\mathrm{J}$, et al. Thymoglobulin induction protects liver allografts from ischemia/ reperfusion injury. Clin Transplant. 2005;19(4)507-11.

12. Mehrabi A, Mood Zh A, Sadeghi M, Schmied BM, Müller SA, Welsch Th, et al. Thymoglobulin and ischemia reperfusion injury in kidney and liver transplantation. Nephrol Dial Transplant. 2007;22 [Suppl 8]:viii54-viii60.

13. Issa NC, Fishman JA. Infectious complications of antilymphocyte therapies in solid organ transplantation. Clin Infect Dis. 2009;48(6):772-86.
14. Adu D, Cockwell P, Ives NJ, Shaw D, Wheatley K. Interleukin-2 receptor monoclonal antibodies in renal transplantation: meta-analysis of randomised trials. BMJ. 2003;326(7393):789-93.

15. Keown P, Balshaw R, Khorasheh S, Chong M, Marra C, Kalo Z, et al. Metaanalysis of basiliximab for immunoprophylaxis in renal transplantation. Biodrugs. 2003;17(4):271-9.

16. Webster AC, Playford EG, Higgins G, Chapman JR, Craig JC. Interleukin 2 receptor antagonists for renal transplant recipients: a meta-analysis of randomized trials. Transplantation. 2004;77(2):166-76.

17. Birkeland SA. Steroid-free immunosuppression in renal transplantation: a long-term follow-up of 100 consecutive patients. Transplantation. 2001;71(8)1089-90.

18. Peters ThG, Shaver TR, Ames JE 4th, Santiago-Delpin EA, Jones KW, Blanton JW1. Cold ischemia and outcome in 17.937 cadaveric kidney transplants. Transplantation. 1995;59(1):191-6.

19. Lebranchu Y, Bridoux F, Buchler M, Le Meur Y, Etienne I, Toupance O, et al. Immunoprophylaxis with basiliximab compared with antithymocyte globulin in renal transplant patients receiving MMF-containing triple therapy. Am J Transplant. 2002;2(1):48-56

20. Mourad G, Rostaing L, Legendre C, et al. Sequential protocols using basiliximab versus antithymocyte globulins in renal-transplant patients receiving mycophenolate mofetil and steroids. Transplantation. 2004;78(4):584-90.

21. Ciancio G, Burke GW, Gaynor JJ, Roth D, Kupin W, Rosen A, et al. A randomized trial of thymoglobulin vs. alemtuzumab (with lower dose maintenance immunosuppression) vs. daclizumab in renal transplantation at 24 months of follow-up. Clin Transplant. 2008;22(2):200-10.

22. Abou-Ayache R, Buchler M, Lepogamp P, Westeel P, Le Meur Y,Etienne I, et al. CMV infections after two doses of daclizumab versus thymoglobulin in renal transplant patients receiving mycophenolate mofetil, steroids and delayed cyclosporine A. Nephrol Dial Transplant. 2008;23(6):2024-32.

23. Mandelbrot D. Anti-IL-2 receptor antibodies versus anti-thymocyte globulin for induction therapy in kidney transplantation. J Am Soc Nephrol. 2009; 20 (6): 1170-1171

24. Kasiske BL, Zeier MG, Chapman JR, Craig JC, Ekberg H, Garvey CA, et al. KDIGO clinical practice guideline for the care of kidney transplant recipients a summary KDIGO guideline for kidney transplant recipients. Kidney International. 2010;77(2):299-311.

25. Gil J, Sampaio M, Gil JS, Dong J, Kuo HT, Danovicth GM, et al. Induction immunosuppressive therapy in the elderly kidney transplant recipient in the United States. Clin J Am Soc Nephrol. 2011;6(5):1168-78. 